\title{
Importance Et Contributions De La Fete "Wemexwe"' Dans Le Developpement Des Communes De La Basse Vallee De L'oueme
}

\author{
Aklamavo Basile \\ Gonzallo Germain \\ Vigninou Toussaint \\ Laboratoire d'Etudes des Dynamiques Urbaines et Régionales (LEDUR), \\ Département de Géographie et Aménagement du Territoire/ FLASH \\ Université d'Abomey-Calavi (UAC), Cotonou, République du Bénin \\ (Afrique de l'Ouest)
}

doi: 10.19044/esj.2017.v13n26p136 URL:http://dx.doi.org/10.19044/esj.2017.v13n26p136

\begin{abstract}
Faced with the inability of the process of decentralization to achieve true grassroots development in Benin, people have turned towards the endogenous development initiatives. These initiatives refer to the community festivals that existed long before the advent of the decentralization process in Benin. This is a typical case of the "Wémexwé" festival in the lower Ouémé valley. This study aims to analyze the importance of socio-cultural realities in the development of localities. In order to understand the importance of community festivals in the development of local authorities, the documentation of this subject is practically non-existent. The participating observation and the field survey made it possible to collect the necessary data from a sample of 200 people. The sample, however, is composed of local elected representatives, local elites, members of the intermunicipal coordination committee of the "Wémexwe" festival, development associations, religious leaders, people, and the traditional and notable leaders of the lower valley of the Ouémé. Thus, the technique of "snowball" was used as a sampling method. The SWOT model (Strengths, Weakness, Opportunities, Threats) was used to analyze the results. According to this study, the community festival "Wémexwé" is of great importance in the development of local communities in the lower valley of Ouémé. Due to its yearly celebration, it leaves traces of development on the site having sheltered it. This includes socio-communal infrastructures and paths that were arranged and overlaid. It has invested approximately CFAF 246324508 in all the four communes of the lower Ouémé valley from the first edition in 2009 to the sixth edition in 2015.
\end{abstract}


Keywords: "Wémexwé", communal feasts, Low valley of the Ouémé, Decentralization, local Development

\section{Résumé}

Face à l'incapacité du processus de la décentralisation à concrétiser un véritable développement à la base au Bénin, les populations se tournent vers les initiatives endogènes de développement que sont les fêtes communautaires qui existaient bien avant l'avènement du processus de décentralisation au Bénin. C'est le cas typique de la fête "Wémexwé" dans la basse vallée de l'Ouémé, objet de la présente étude qui vise à analyser l'importance des réalités socio-culturelles dans le développement des localités. Pour comprendre l'importance des fêtes communautaires dans le développement des collectivités locales, la documentation en la matière étant pratiquement inexistante, l'observation participante et l'enquête de terrain ont permis de collecter les données nécessaires, auprès d'un échantillon de 200 personnes constituées des élus locaux, des élites locales, des membres du bureau de la coordination intercommunale de la fête "Wémcxwé', des associations de développement, des responsables religieux, des populations, des chefs traditionnels et notables de la basse vallée de l'Ouémé. Ainsi, la technique de "boule de neige" a été utilisée comme méthode d'échantillonnage. Le modèle SWOT (Strengths, Weakness, Opportunities, Threats) a permis d'analyser les résultats obtenus. Il ressort de cette étude que la fête communautaire "Wémexwé" est d'une grande importance dans le développement des collectivités locales de la basse vallée de l'Ouémé. Chaque année de sa célébration, elle laisse des traces de développement sur le site l'ayant abrité : infrastructures sociocommunautaires et voies aménagées et recouvertes de latérite. Elle a permis d'investir environ 246324 508F CFA dans l'ensemble des quatre communes de la basse vallée de l'Ouémé depuis la première édition en 2009 jusqu'à la sixième édition en 2015.

Mots-clés: “Wémcxwé’, fêtes communautaires, Basse vallée de l'Ouémé, Décentralisation, Développement local

\section{Introduction}

Selon Déberre (2007), la décentralisation et le développement local apparaissent comme deux démarches pour construire le développement à la base. Le Bénin a enclenché le processus de la démocratisation depuis l'historique conférence nationale des forces vives de février 1990. Ce nouveau mode de gestion démocratique est soutenu en décembre 2002, par l'avènement de la décentralisation qui vient consacrer le partage du pouvoir, 
des compétences et des moyens entre 1'Etat central et les collectivités territoriales locales. Cependant, dans la pratique, le constat est déplorable, surtout s'agissant de l'effectivité du développement local. Après plus d'une dizaine d'années d'expérimentation de cette réforme de l'administration territoriale au Bénin, force est de constater qu'il y a plus de discours et théories politiques que d'actions et de pratiques de développement à la base.

$\mathrm{Au}$ demeurant, le processus de la décentralisation peine à concrétiser un véritable développement local dans les collectivités locales pour plusieurs raisons, notamment la politisation à outrance, la gestion peu orthodoxe des affaires locales, la non-maîtrise des bases et principes de la décentralisation par certains élus locaux, l'incompétence de certains cadres administratifs nommés sur les bases politiques et partisanes, la mauvaise gouvernance locale, etc. Zannou (2014) montre que le comportement des principaux acteurs du développement et les insuffisances relatives aux textes de la décentralisation ne favorisent pas une bonne gouvernance locale. Ces maux minent dangereusement la gestion des collectivités locales et hypothèquent leur développement.

La question qui se dégage de ce constat est celle de savoir si la décentralisation assure réellement le développement local dans nos communes. Piveteau (2005) va dans la même direction d'analyse de l'effectivité du développement local en s'interrogeant : "La décentralisation en Afrique de l'ouest facilite-t-elle le développement local ?». Pour Totté et al. (2003), la politique en Afrique de l'Ouest, outrepassant ses limites, a vidé la décentralisation de son sens originel de développement à la base, au point que, de leur côté, Dubresson et al. (2005) trouvent, d'après leur analyse, qu'il faut repenser le lien entre la décentralisation et le développement local.

Face à l'échec de la politique de décentralisation dont les acteurs sont plus préoccupés par le souci du contrôle des municipalités que du développement local, les populations, soucieuses du développement de leur localité, initient de nouvelles dynamiques de développement à la base, dont les fêtes communautaires qui se veulent apolitiques. Ces initiatives locales, en pleine expansion, tendent à se généraliser sur l'ensemble du territoire national, supplantant ainsi le processus de la décentralisation sur le terrain. C'est dans cette nouvelle vague de dynamiques endogènes de développement que s'inscrit la fête communautaire des "Wémsnu' dite 'Wémcxwé".

\section{Matériels et Méthodes \\ Matériels}

Etabli dans la basse vallée de l'Ouémé et sur le plateau de SakétéPobè, le pays "Wéme" est un espace géographique situé au sud-est de la République du Bénin. Avec une superficie de $810 \mathrm{~km}^{2}$ environ, il s'étend sur une grande partie du département de l'Ouémé. Il est limité au nord par le 
département du Zou, à l'est par la lagune de Porto-Novo, les communes de Porto-Novo, d'Akpro-Missérété et le département du Plateau, au sud par le lac Nokoué et la commune de Sèmè-Kpodji et à l'ouest par le département de l'Atlantique et le lac Nokoué.

Le pays 'Wéme" est localisé entre $6^{\circ} 24$ ' et $6^{\circ} 58^{\prime}$ latitude Nord et entre $2^{\circ} 24^{\prime}$ et $2^{\circ} 35^{\prime}$ longitude Est. Il regroupe les communes d'Adjohoun, des Aguégués, de Bonou et de Dangbo. Les caractéristiques naturelles de la région font de celle-ci une zone favorable à l'implantation humaine. La Figure 1 montre la situation administrative du cadre d'étude.

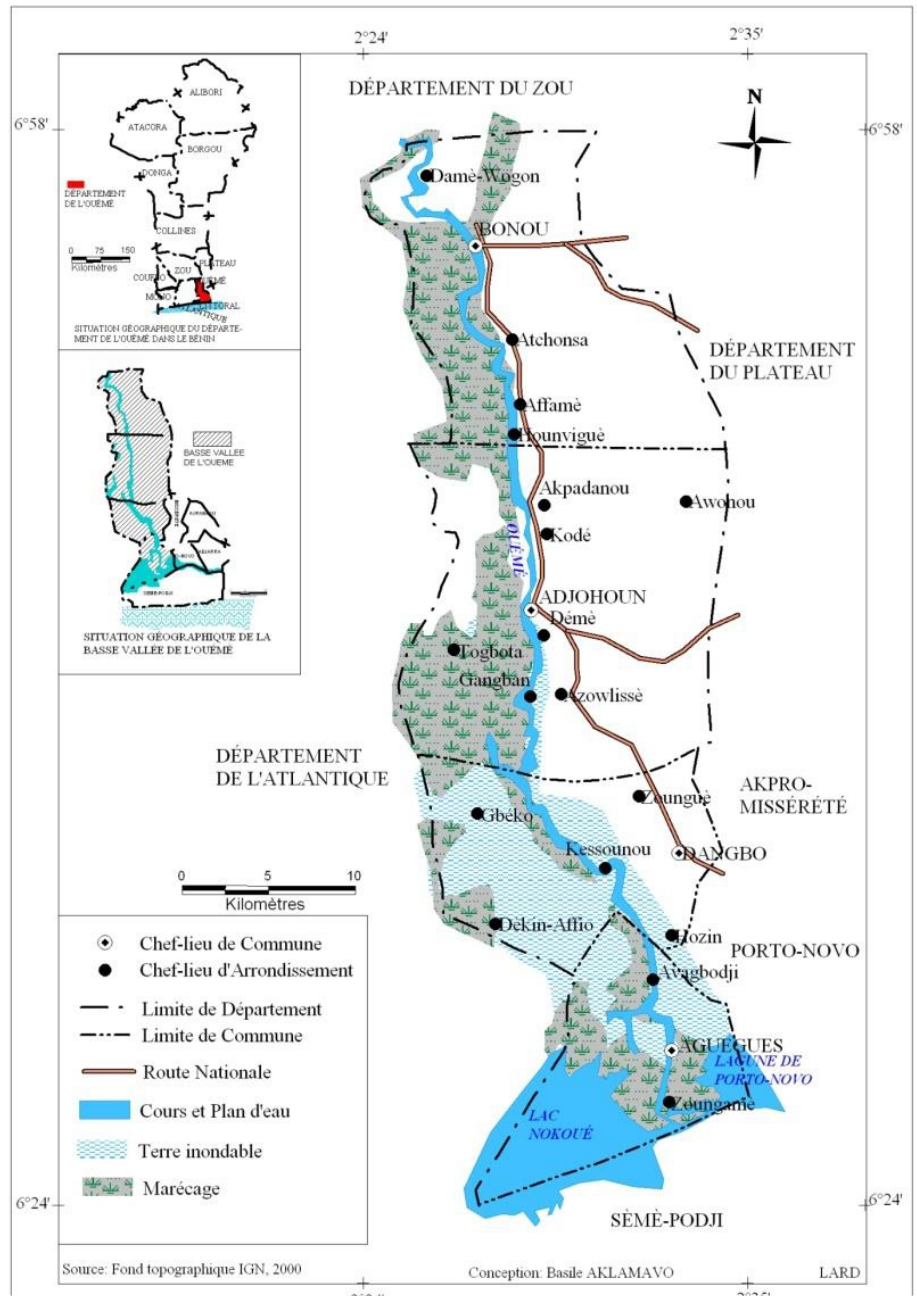

Figure 1. Situation géographique du secteur d'étude

Le pays "Wéme" offre nombreuses conditions naturelles favorables à la production. Le climat subéquatorial à deux saisons de pluie est un 
élément régulateur de la vie agricole des "Wémenu". De par sa position géographique, le pays "Wéme" bénéficie d'une humidité permanente, un facteur très favorable à la croissance des végétaux. Le fleuve Ouémé, le plus important cours d'eau du réseau hydrographique béninois, et la rivière Togbo, apparaissent comme des conditions favorables aux activités économiques du secteur primaire dans le pays "Wéme". Les sols sont très fertiles grâce au dépôt de limon et des matières organiques charriés lors des crues du fleuve Ouémé. Les périodes des crues sont les plus souhaitées et les plus attendues par les paysans. De l'amplitude des eaux dépend une bonne ou mauvaise saison agricole. Le transport des alluvions fertilisantes, depuis les hautes altitudes du bassin fluvial, est d'autant plus important que le volume d'eau écoulée est élevé.

Les activités menées par les "Wémenu'sont multiples. L'agriculture est la plus importante et occupe la plus grande frange de la population. Cette une activité surtout réservée aux hommes, les femmes y participent surtout pendant les semailles et les récoltes. Certains paysans se regroupent en coopératives agricoles, mais la grande majorité préfère une exploitation individuelle de la terre. Quelques techniciens agricoles et de rares fermiers pratiquent cette activité (Hounsinou, 2007).

Selon les travaux de Hounsinou (2007), le pays "Wéme" seul fournit plus de $80 \%$ de la production agricole du département de l'Ouémé. Cette production est diversifiée. Nous avons:

le maïs qui est le plus important produit vivrier et le plus transformé dans l'Ouémé;

- $\quad$ Le manioc, la deuxième culture après le maïs;

- $\quad$ Le niébé, le seul légumineux cultivé dans le département; La patate douce

- $\quad$ Les cultures maraîchères et les oléagineux;

- Le taro, la banane plantain, l'orange, la mandarine la papaye, la mangue, du sésame, l'arachide, etc.

Par ailleurs, dans le domaine de la pêche, le fleuve Ouémé et la rivière TJgbo fournissent une grande variété de produits halieutiques, surtout des poissons. Les écrevisses, les crevettes, les crabes sont d'autres genres de produits de pêche rencontrés dans le pays "Wéme". La pêche se pratique beaucoup plus dans la commune des Aguégués qui ne dispose pas d'une grande étendue de terres cultivables (environ $52 \mathrm{~km}^{2} \operatorname{sur} 103 \mathrm{~km}^{2}$ ).

D'autres activités économiques occupent aussi les "Wémsnu'. Il s'agit des activités d'élevage du gros et de petits bétails (les bovins, les caprins et les porcins.

L'artisanat est aussi développé dans le pays "Wéme". On distingue : la forge, la vannerie, le tissage, la menuiserie, la maçonnerie. 
S'agissant des activités commerciales, les échanges se font dans les marchés comme les marchés de Dangbo, de Hozin, d'Azowlissè, de Tatonnonkon, etc. Les transactions se font aussi à travers les cours d'eau (le fleuve Ouémé, la rivière Sô, la lagune de Porto-Novo, ...) qui constituent les voies de communication et de transports des produits vivriers et halieutiques vers d'autres communes de l'Ouémé, de l'Atlantique et du Zou. En effet, le transport fluvial, terrestre est développé et joue un rôle important dans la régulation des activités à "Wéme".

De nos jours, l'activité d'exploitation des carrières de sable lagunaire ou fluvial occupe les populations du pays "Wéme". On distingue les carrières d'exploitation de sable fluvial d'Adjohoun, de Bonou, de Dangbo et des Aguégués. Cette activité est menée aussi bien par les hommes que les femmes, et même par les jeunes gens déscolarisés.

\section{Méthodes}

La démarche méthodologique adoptée dans le cadre de cette étude est celle propre à la recherche en sciences sociales comme la sociologie. Les fêtes communautaires sont des faits sociaux qui entrainent des changements sociaux. Durkheim (1894) affirme qu'il faut «traiter les faits sociaux comme des choses $\gg$.

La méthode de recherche utilisée s'articule autour de la recherche documentaire, des techniques et outils de collecte des données, de la détermination du groupe cible et de l'échantillonnage, des techniques de traitement des données et d'analyse des résultats.

- Recherche documentaire a permis de consulter des thèses, des mémoires, des revues et articles traitant des questions de la décentralisation et du développement local.

- L'observation participante, l'enquête de terrain et l'entretien ont été les techniques utilisées pour la collecte des données.

En effet, l'observation participante a permis de participer activement à la fête, d'observer les rituels de la célébration de la fête et des séquences de réjouissances, de procéder à la visite des stands de la foire de "Wémexwé" et d'apprécier les prestiges culturelles, artisanales et culinaires du pays Wéms et de visiter les infrastructures socio-communautaires réalisées grâce à la fête 'Wémexwé" depuis sa création en 2010 jusqu'à la 6 ème édition en 2015.

S'agissant des enquêtes de terrain, elles ont permis de collecter les données relatives aux potentialités de la basse vallée de l'Ouémé, aux aspects socio-culturels du développement local que véhicule la fête "Wémexwé" et à l'importance et aux contributions de la fête "Wémexwé" dans le développement de la basse vallée de l'Ouémé. Au cours de ces enquêtes, les 
questionnaires élaborés ont été administrés aux personnes sélectionnées selon un critère d'échantillonnage bien défini.

Quant à l'entretien, il est réalisé au moyen d'un guide d'entretien avec les maires des communes de la basse vallée de l'Ouémé, avec les élites locales, les notables et les têtes couronnées. L'entretien a porté sur la connaissance du cadre d'étude, le développement local, la décentralisation et l'historique de la fête "Wémexwé" et ses contributions dans le développement des communes de la basse vallée de l'Ouémé.

- $\quad$ Les outils de collecte de données sont:

Les fiches d'enquêtes et les guides d'entretien qui ont permis de collecter les données géographiques, démographiques, socioculturelles, économiques, les données sur l'historique de la fête "Wémexwé" et sur les contributions de ladite fête au développement.

Les données collectées sont d'ordres qualitatif et quantitatif. Les données qualitatives concernent la perception et l'appréciation, par les populations et autres acteurs du développement de la Basse Vallée de l'Ouémé, de l'importance de la fête "Wémexwé". Les données quantitatives concernent l'évaluation des contributions de cette fête communautaire dans le développement local dans la Basse Vallée de l'Ouémé.

A propos des groupes cibles et de l'échantillonnage, au regard de la diversité des informations à recueillir, il est considéré comme population d'étude l'ensemble des acteurs impliqués dans la vie socio-culturelle, économique et communautaire de la basse vallée de l'Ouémé. Des personnes ressources capables de fournir des informations ont été interrogées. A cet effet, les groupes cibles sont constitués des élus locaux, les élites locales, les membres du bureau de la coordination intercommunale de la fête "Wémexwé", les associations de développement, les responsables religieux, les populations, les chefs traditionnels et notables de la basse vallée de l'Ouémé. Ainsi, la technique de "boule de neige" a été utilisée. Cette technique a permis de collecter les informations auprès d'un nombre représentatif de 200 personnes de différentes catégories socioprofessionnelles (Tableau I). 
Tableau I. Personnes enquêtées

\begin{tabular}{|c|c|c|c|c|c|}
\hline $\begin{array}{c}\text { Catégories socio- } \\
\text { Professionnelles }\end{array}$ & \multicolumn{3}{|c|}{ Effectifs par Commune } & Total \\
\cline { 2 - 5 } & Adjohoun & Aguégués & Bonou & Dangbo & \\
\hline Elus locaux & 05 & 05 & 05 & 05 & 20 \\
\hline Elites locales & 15 & 10 & 10 & 15 & 50 \\
\hline $\begin{array}{c}\text { Membres du bureau } \\
\text { de la coordination } \\
\text { intercommunale de la } \\
\text { fête "Wémexwé" }\end{array}$ & 01 & 01 & 01 & 02 & 05 \\
\hline $\begin{array}{c}\text { Associations de } \\
\text { développement, }\end{array}$ & 05 & 04 & 04 & 06 & 19 \\
\hline $\begin{array}{c}\text { Responsables } \\
\text { religieux, }\end{array}$ & 02 & 01 & 01 & 02 & 06 \\
\hline Populations & 20 & 20 & 20 & 20 & 80 \\
\hline $\begin{array}{c}\text { Chefs traditionnels et } \\
\text { notables de la basse } \\
\text { vallée de l'Ouémé }\end{array}$ & 05 & 05 & 05 & 05 & 20 \\
\hline \begin{tabular}{c} 
Total : \\
\hline
\end{tabular} & 53 & 46 & 46 & 55 & 200 \\
\hline
\end{tabular}

Source : Travaux de terrain (août, 2015)

Les résultats ont été analysés sur la base du modèle SWOT (Strengths, Weakness, Opportunities and Threats). Ce modèle a permis de faire l'analyse systémique des forces, faiblesses, opportunités et menaces qui influencent la fête "Wémexwé" dans le développement local dans la basse vallée de l'Ouémé. Le but de l'analyse des données des travaux de terrain sur la base du modèle SWOT est de prendre en considération ces facteurs internes et externes, dans la stratégie d'étude de l'importance des fêtes communautaires dans le développement local, pour maximiser les potentiels les forces et les opportunités et minimiser les effets des faiblesses et des menaces pour la survie et la meilleure évolution de la fête "Wémexwé".

\section{Résultats et Discussion}

\section{Résultats}

Les fêtes communautaires sont d'une grande importance dans le développement des collectivités locales par le fait qu'elles font participer les populations locales aux actions de développement de leurs localités et assurent le renforcement de la cohésion sous-régionale entre les communes de la Basse Vallée de l'Ouémé.

En effet, cette fête "Wémexwé"'est initiée sur fond de spiritualité liée à la mémoire du Feu Révérend Père Dominique ADEYEMI qui avait œuvré, de par le passé, au développement spirituel et socio-économique de la basse vallée de l'Ouémé.

Aussi chaque célébration de "Wémexwé" laisse-t-elle des traces de développement sur le site l'ayant abrité: infrastructures sociocommunautaires, voies aménagées, etc. 


\section{Impacts de la fête communautaire "Wémcxwé", sur le développement local de la vallée de l'Ouémé}

Les impacts de la "Wémexwé", sur le développement des communes de la basse vallée de l'Ouémé sont de divers ordres.

\section{$>\quad$ Les réalisations de la fête communautaire "Wémcxwé",}

Dans un élan de fraternité et de solidarité, les filles et fils des communes d'Adjohoun, des Aguégués, de Bonou et de Dangbo ont donné la preuve que le développement de leur localité dépend d'eux. Car, cette fête, depuis la première édition d'origine qui a lieu à Azowlissè (commune d'Adjohoun) en 2010 à la sixième édition aux Aguégués en 2015, permet à chaque localité qui abrite la fête de bénéficier des infrastructures sociocommunautaires. Les photographies ci-dessous en montrent deux exemples.

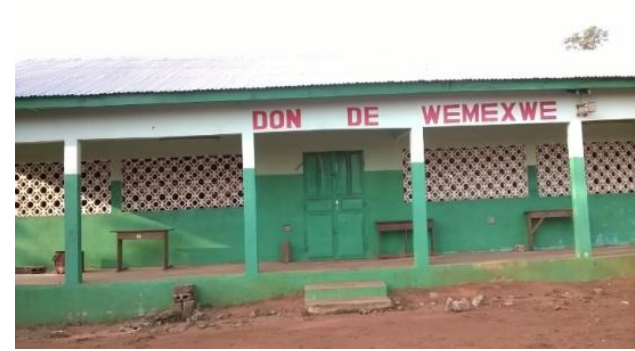

Photo 1. Une salle de classe construite par les organisateurs de "Wémexwé", au CEG d'Azowlissè.

Prise de vue : Aklamavo (septembre 2015)

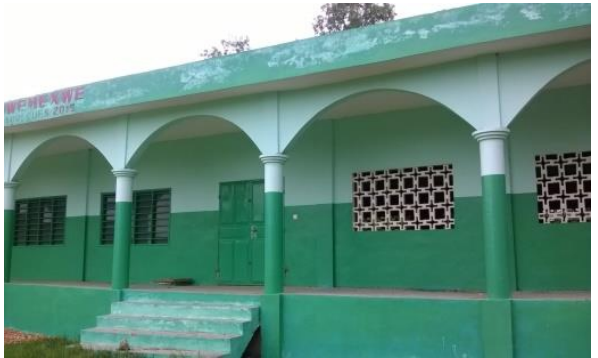

Photo 2. Un laboratoire moderne et une salle de classe entièrement équipés, construits par les organisateurs de 'Wémexwé" au CEG Avagbodji.

Prise de vue : Aklamavo (septembre 2015)

Le Tableau II présente l'état récapitulatif des réalisations de "Wémexwé" depuis la première édition jusqu'à la sixième. 
Tableau II. Récapitulatif des réalisations de 'Wémexwé',

\begin{tabular}{|c|c|c|c|c|}
\hline Communes & $\begin{array}{l}\mathrm{N}^{\circ} \\
\text { d'édition/ } \\
\text { Année }\end{array}$ & Réalisations de "Wémexwé" & $\begin{array}{l}\text { Coût des } \\
\text { réalisations } \\
\text { (CFA) }\end{array}$ & Total (FCFA) \\
\hline \multirow{4}{*}{ Adjohoun } & $1^{\text {ère }}: 2010$ & Néant & Néant & \multirow{4}{*}{44246150} \\
\hline & \multirow{3}{*}{$5^{\text {ème }}: 2014$} & $\begin{array}{l}\text { Construction d'une salle de } \\
\text { classe au CEG d'Adjohoun }\end{array}$ & 6858562 & \\
\hline & & $\begin{array}{l}\text { Construction d'une salle de classe } \\
\text { au CEG d'Azowlissè }\end{array}$ & 7362002 & \\
\hline & & $\begin{array}{l}\text { Construction d'un module de trois } \\
\text { classes avec le bureau de la } \\
\text { Direction au CEG de Goutin }\end{array}$ & 30025586 & \\
\hline \multirow[t]{2}{*}{ Aguégués } & $2^{\text {ème }}: 2011$ & $\begin{array}{l}\text { Voie Aménagée recouverte de } \\
\text { latérite, reliant les arrondissements } \\
\text { d'Avagbodji et des Aguégués }\end{array}$ & 78569445 & \multirow[t]{2}{*}{114096301} \\
\hline & $6^{\text {ème }}: 2015$ & $\begin{array}{l}\text { Construction d'un laboratoire de } \\
\text { type moderne et d'une salle de } \\
\text { classe au CEG Avagbodji }\end{array}$ & 35526856 & \\
\hline \multirow[t]{2}{*}{ Bonou } & \multirow[t]{2}{*}{$3^{\text {ème }}: 2012$} & $\begin{array}{l}\text { Construction d'un module de trois } \\
\text { classes au CEG Affamey }\end{array}$ & 32632745 & \multirow[t]{2}{*}{34889032} \\
\hline & & $\begin{array}{c}\text { Construction d'une latrine à trois } \\
\text { cabines au CEG d'Affamey }\end{array}$ & 2256287 & \\
\hline \multirow[t]{2}{*}{ Dangbo } & \multirow[t]{2}{*}{$4^{\text {ème }}: 2013$} & $\begin{array}{c}\text { Construction d'un module de trois } \\
\text { classes au CEG } 2 \text { de Dangbo }\end{array}$ & 33569451 & \multirow[t]{2}{*}{53093025} \\
\hline & & $\begin{array}{c}\text { Construction de laboratoire au } \\
\text { CEG Zounguè }\end{array}$ & 19523574 & \\
\hline TOT & & & & 246324508 \\
\hline
\end{tabular}

Source : Travaux de terrain septembre 2015

L'observation du tableau II permet de relever que:

$\checkmark \quad$ La première édition de "Wémexwé" n'a pas connu de réalisations d'infrastructures sociocommunautaires, car elle a servi de base de réflexion pour la définition des objectifs à assigner à cette nouvelle institution sociale qui venait de naître.

$\checkmark \quad$ Les valeurs des réalisations de "Wémexwé" varient, allant croissantes, d'une édition à une autre, d'une commune à une autre. Cette variation de coûts est due à l'ampleur de mobilisation des ressources, qui dépend de la masse contributive, sans cesse croissante, des différents acteurs participants à chaque édition de célébration. Ceci témoigne de la croissance des investissements de"Wémexwé" au fur et à mesure que les éditions s'alternent et se succèdent au fil des années.

$\checkmark \quad$ Le compactage de la voie recouverte de latérite, d'environ 14 kilomètres, reliant les arrondissements d'Avagbodji et des Aguégués, a été financé par le Gouvernement béninois à travers le Ministère des Travaux Publics et des Transports, sous l'impulsion des organisateurs de "Wémexwé". 
Figure 2. Répartition spatiale des infrastructures socio-communautaires réalisées grâce à la fête Wémexwé

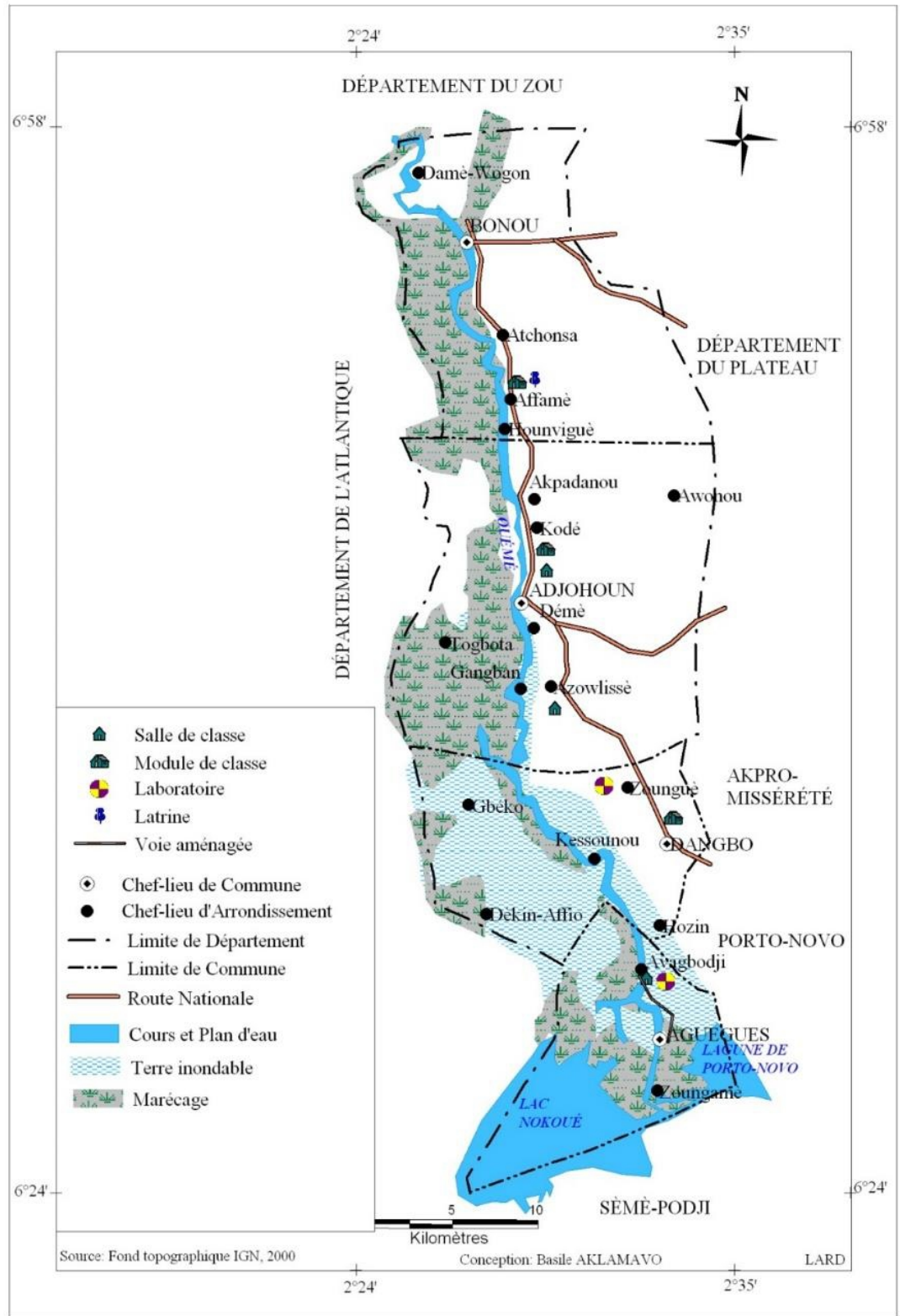

La Figure 2 montre la répartition spatiale des réalisations de "Wémexwé", sur l'ensemble du territoire du pays "Wéme".

\section{Stratégies de mobilisation des ressources de "Wémexwé",}

Les ressources de "Wémexwé', sont de divers ordres. Il s'agit des ressources matérielles issues de dons en nature, des ressources humaines et techniques exploitées dans la réalisation des infrastructures 
sociocommunautaires par la mise à disposition des techniques professionnelles d'ouvrages et de force physique de travail, des ressources financières issues de diverses sources dont les souscriptions, les cérémonies d'appel de fonds, les divers dons volontaires, les cotisations, la vente du tissus de "Wémexwé", etc.

Diverses stratégies sont mises en œuvre par les organisateurs de la fête "Wémexwé" pour mobiliser les ressources servant à concrétiser les réalisations d'infrastructures sociocommunautaires dans les communes de la basse vallée de l'Ouémé. Ces stratégies sont de diverses formes, notamment: - Le mutuel de membres actifs de "Wémexwé": chaque membre a une carte de membre et doit cotiser une somme de douze mille (12000) francs CFA par an, à raison de mille (1000) francs CFA par mois.

Les souscriptions volontaires des natifs de la basse vallée de l'Ouémé, des partenaires et de la diaspora.

- La vente de tissu de "Wémexwé" dont le prix est majoré d'un surplus à défalquer pour alimenter les caisses de fonds de réalisations.

- $\quad$ La participation financière des mairies des quatre communes de la basse vallée de l'Ouémé.

- La cérémonie d'appel de fonds comparable à la technique de mobilisation de fonds appelée “'Zindo" organisée dans les régions Fon du plateau d'Abomey. Cette cérémonie dite cérémonie de lancement officiel des festivités de la nouvelle édition de la fête "Wémexwé", a lieu trois (03) mois avant la nouvelle édition (généralement dans le mois de septembre précédant cette édition), dans la commune devant abriter ladite édition. Ce creuset permet de collecter les libéralités, les promesses de dons, de vendre le tissu de "Wémexwé" et de mobiliser les populations et les préparer à accueillir, dans une attente certaine, la nouvelle édition prochaine.

\section{Aspects socio-culturels de développement de la fête communautaire" Wémexwé",}

Les aspects socio-culturels de développement ressortis de la fête communautaire "Wémexwé" sont de divers ordres. Ils révèlent l'importance capitale de cette fête dans le développement socio-économique et communautaire de l'ensemble des communes de la basse vallée de l'Ouémé.

\section{$\checkmark \quad$ Participation et implication des populations au processus de développement local}

L'un des principes fondamentaux du processus de développement local est la participation et l'implication des populations dans les affaires de développement de leur localité. Parallèlement au processus de la décentralisation, la fête communautaire "Wémexwé"permet aujourd'hui aux populations natives de la basse vallée de l'Ouémé de s'impliquer et de participer aux affaires de développement de leur localité. Elles se sentent 
beaucoup plus concernées par le développement de leur terroir. Pour elles, «c'est le fils qui bâtit la cité de ses pères ». La fête "Wémexwé"'est un atout incontournable pour le développement effectif de la basse vallée de l'Ouémé. La participation et l'implication des populations locales de la basse vallée de l'Ouémé est le premier aspect de développement contenu dans l'organisation de la fête "Wémexwé".

\section{$\checkmark \quad$ Réalisation de coopération entre les communes de la basse vallée de l'Ouémé}

La fête "Wémexwé" a permis aux quatre communes de la basse vallée de l'Ouémé de fédérer leurs énergies et de coopérer pour le développement commun de toute la région. Ceci fait ressortir l'aspect d'une intercommunalité agissante entre ces communes qui partagent les mêmes réalités culturelles et sociolinguistiques et des terroirs aux caractéristiques physiques, naturelles et géographiques identiques. L'aménagement de la basse vallée de l'Ouémé appartenant aux quatre collectivités territoriales accroit substantiellement la production céréalière dans cette zone et contribue à l'autosuffisance alimentaire des populations. Par conséquent, ces communes de la basse vallée de l'Ouémé doivent inéluctablement, pour se développer, fondre leurs énergies créatrices individuelles dans une dynamique de coopération intercommunale. Et la fête "Wémexwé" est un moyen le plus adéquat pour atteindre cette collaboration entre les communes de la basse vallée de l'Ouémé. En somme, la fête "Wémexwé' 'est l'origine de la coopération intercommunale pour le développement de la basse vallée de l'Ouémé.

\section{$\checkmark \quad$ Contributions des relations au développement de la basse vallée de l'Ouémé}

La réputation de la fête"Wémexwé"ne regroupe pas seulement les fils et filles de la basse vallée. Elle est aussi l'occasion de faire participer les amis des natifs de la basse vallée, sympathisants, les invités de toutes les régions du Bénin et du monde, les touristes, etc. Chaque catégorie de personnes assistant à la célébration de la fête, manifestations, participe aux réalisations de la fête par leurs divers dons, leurs béatitudes, leurs souscriptions. Aussi, les natifs de la basse vallée de l'Ouémé mettent-ils à contribution leurs relations à l'échelle nationale et internationale pour le développement de leur région.

De tout ce qui précède, c'est à juste titre d'affirmer que la fête "Wémexwé"se présente comme l'occasion pour célébrer les relations partenariales et de les mettre à contribution et au service du développement de la basse vallée de l'Ouémé. 


\section{$\checkmark \quad$ Collaboration et complémentarité entre institution publique (Conseil communal) et institution sociale (le "Wémعxwé') pour le développement}

Selon les résultats des travaux de terrain, les autorités locales des municipalités de la basse vallée de l'Ouémé impliquent à certaines séances de travail ou sessions ordinaires, les membres du Bureau de la coordination intercommunale de "Wémcxwé", en vue d'échanger les opinions et avis allant dans le sens du développement des communes de la basse vallée de l'Ouémé. Aussi, ces élus locaux participent-ils tant financièrement que matériellement aux réalisations de la fête "Wémcxwé'. Ils leur présentent les projets de développement communal de leurs localités et les activités à y mener. Ainsi, les organisateurs de la fête "Wémcxwé" s'en servent pour planifier leurs réalisations dans ces localités. Cette méthode de collaboration et de coopération entre l'institution sociale "Wémexwé" et les conseils communaux de la basse vallée de l'Ouémé, est salutaire pour un développement local harmonieux et constitue une forme de participation de la population à l'élaboration et à la mise en œuvre des projets et des plans communaux de développement dans la basse vallée de l'Ouémé.

La collaboration et la complémentarité entre les élus locaux et l'institution sociale, le "Wémexwé', sont une expérience louable à retenir, à encourager, à promouvoir et à pérenniser pour un réel développement futur des communes de la basse vallée de l'Ouémé.

\section{de l'Ouémé}

Par affirmation identitaire, entendons un sentiment d'attachement passionné à ce qui constitue le caractère singulier d'un groupe social ou d'une communauté ayant une réalité culturelle commune et un terroir bien déterminé.

A cet égard, la fête" "Wémexwé" génère déjà chez les "Wémsnu" un sentiment de fierté d'être natifs d'une région du sud du Bénin, cette basse vallée réputée la plus riche du monde après celle du Nil, cette basse vallée qui est capable de nourrir tous les béninois si elle était exploitée à bon escient. Déjà, ils se réclament de la basse vallée et défendent leur culture et leur dialecte, jadis considérés comme rétrogrades.

Par la fête "Wémexwé", les filles et fils de la basse vallée de l'Ouémé ont retrouvé leur identité culturelle. Chacun veut apporter sa pierre à l'édifice de la reconstruction du développement du pays "Wéme". Ainsi, ce sentiment d'affirmation identitaire participe, à grande part, au développement de la basse vallée de l'Ouémé. 


\section{Perspectives de la fête communautaire "WémExwé"}

En guise de perspectives, les résultats du forum sur le développement de la basse vallée de l'Ouémé sont porteurs d'avenir meilleur pour le pays "Wéme". Les organisateurs de cette fête ne comptent pas s'arrêter en un si bon chemin. Tous les domaines de la vie de cette région seront exploités : l'agriculture, l'économie, la formation des jeunes, l'alphabétisation sont autant de centres d'intérêts les plus importants à développer. Beaucoup d'actions seront concrétisées sur le terrain, comme le déclare un natif du pays "Wéme" lors de la célébration de la 6 ème édition de fête de Wémexwé", dans la commune des Aguégués : "De plus rien de beau, de grand et de durable ne s'est jamais fait et ne se fera jamais dans la facilité. La route qui mène au véritable développement est longue, difficile, "boueuse" et souvent ingrate. Mais des notes rassurantes, pleines d'espoir et d'espérance, nous parviennent de la commune d'Adjohoun, des initiateurs du projet intercommunal Songhaï et des organisateurs du Forum pour le développement de la basse vallée. Ils nous parlent un nouveau langage, d'une autre manière de concevoir et d'asseoir une véritable dynamique de développement rural »

Les résultats de cette étude ont été analysés sur la base du modèle d'analyse SWOT (Strengths, Weakness, Opportunities and Threats). La Figure 3 présente la substance de cette analyse. 


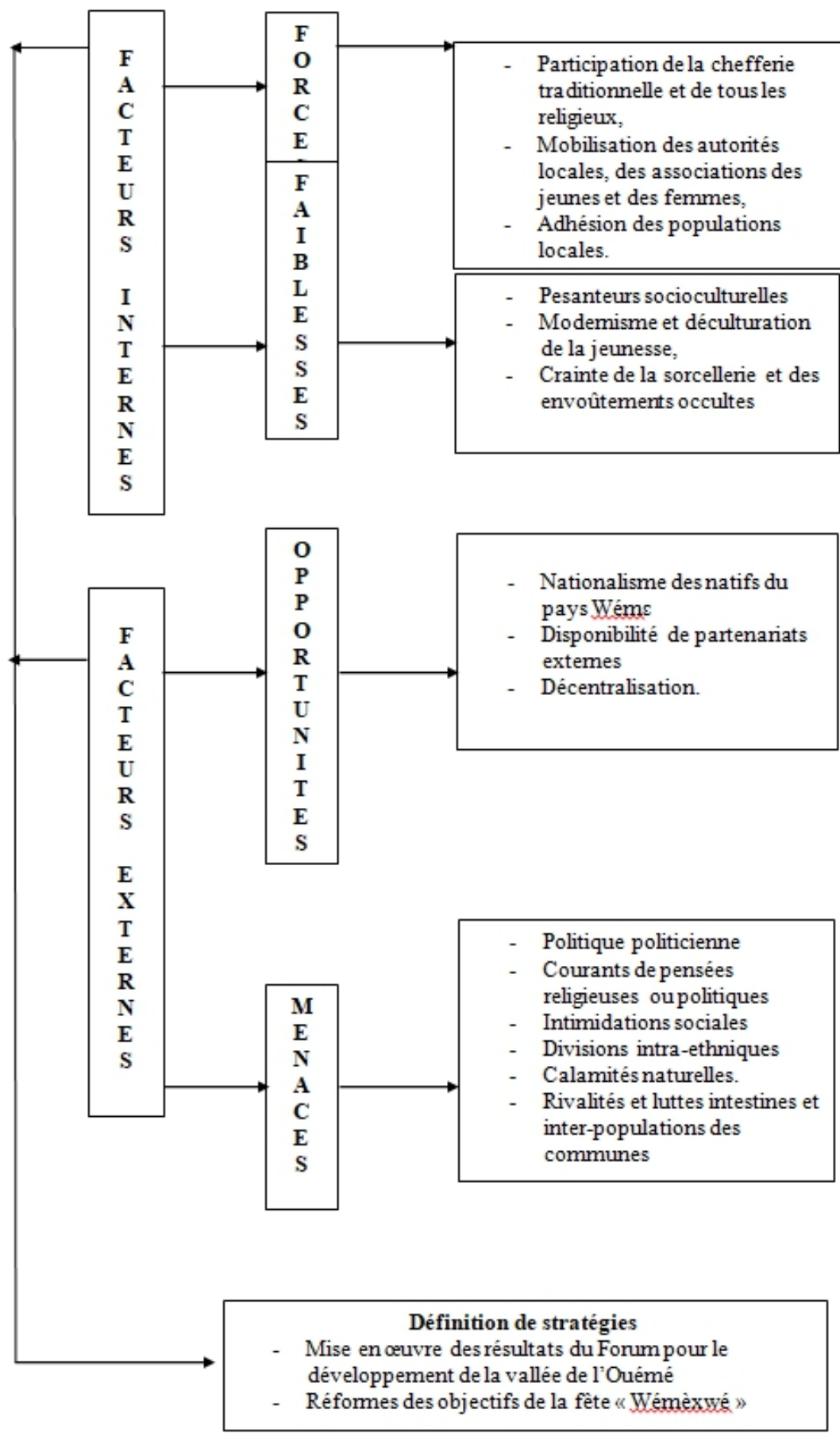

Figure 3. Modèle d'analyse de l'importance et des impacts de la fête communautaire « Wémexwé » sur le développement local dans le pays wéme, à l'aide de (SWOT)

Source : Inspiré de Yabi (2004) 
La lecture de la Figure 3 permet de mettre en lumière les facteurs internes (forces et faiblesses) et les facteurs externes (opportunités et menaces) qui interagissent et influent sur la vie et l'évolution de la fête "Wémexwé".

Les forces internes (Mobilisation des autorités locales, adhésion des populations locales, etc.) sont des facteurs qui favorisent beaucoup es regroupements entre les filles et fils d'une communauté, d'une région ou d'un territoire socio-culturel donné pour l'organisation de fêtes de retrouvailles.

Les faiblesses (les pesanteurs socioculturelles, le modernisme et la déculturation de la jeunesse, etc.) sont à la base du désintéressement de certains membres d'une communauté socio-linguistique aux initiatives de retrouvailles. Du coup, ils ne participent pas à l'organisation, ni aux réalisations générées par les fêtes organisées.

Les opportunités offrent les conditions d'une bonne évolution des fêtes communautaires. Dans le cas d'espèce, le processus de la décentralisation en cours au Bénin, le sentiment nationaliste, la disponibilité de partenariat sont autant d'opportunités dont bénéficie la fête "Wémexwé".

Situées en opposition aux opportunités, les menaces sont des facteurs externes qui agissent dans le sens de la destruction des initiatives de regroupement de retrouvailles. En effet, la politique politicienne, les divisions internes au sein d'une communauté, l'esprit de partie, etc. sont des menaces qui détruisent les initiatives de regroupement et entravent la meilleure évolution des fêtes communautaires. La fête "Wémcxwé" est actuellement confrontée à ces menaces.

\section{Discussions}

L'expérience de développement à la base faite par les populations de la basse vallée de l'Ouémé à travers la fête "Wémcxwé' témoigne fort de l'importance de l'implication et de la participation des populations locales dans la prise de décisions et dans la mise en œuvre des projets de développement de leur terroirs. Ainsi, cette fête a permis de rassembler tous les filles et fils de la basse vallée de l'Ouémé autour des idéaux de développement à travers les dons et libéralités, la force de travail.

Cet aspect de développement est relaté par Lafia (1997) qui parle de « dons » pendant la "Gaani', «Mieux, la "Gaani'” est une fête de dons. Les nombreux efforts consentis par les acteurs de la "Gaani" (tant dans la production agricole, artisanale que dans le commerce pour multiplier les richesses) pendant toute une année sont engloutis dans la fête. Les motivations des dons (dépenses qu'ils engendrent) sont diverses : prestiges, générosité, honneur, gratifications psychologiques. Ces motivations sont souvent cachées sous le prétexte d'une obligation morale et impliquent des 
déviations inévitables d'argent. Ces déviations sont révélatrices des véritables intentions des donateurs» (Lafia, 1997).

Par ailleurs, selon les travaux de recherche de Lafia (1997), la "Gaani' est une institution sociale que l'on retrouve chez certains peuples de l'Afrique de l'ouest et même au-delà. Certes, la dénomination varie en fonction des pays. Mais la "Gaani" se célèbre à la Mecque (qui serait, selon certaines hypothèses, le berceau du peuple Batonu) et dans les pays historiquement hostiles à la domination islamique. Il s'agit du BurkinaFaso, du Tchad, du Nigeria (Bussa, Sandiro, Okuta), du Bénin (Nikki, Kouandé, Bouwè, Kandi, Kérou, ...). La "Gaani" est un rendez-vous coutumier visant la cohésion du groupe Batonu. Elle est rendez-vous de donner et de recevoir dont les échos débordent les frontières du Bénin. La "Gaani' est une mise en sciènes des participants. Sa particularité réside dans la cavalcade, les sacrifices, les rites de passage, les dons et contredons, les échanges par lesquels le groupe Batonu vit plus intensément. Elle a lieu après la fête de "Donkonru" "ffête du feu où les gens de lancent le feu, le feu ayant pour ce peuple un pouvoir purificateur. Les jets ou lancement de feu éloignent les mauvais esprits et le malheur que pourrait amener la nouvelle année (Lafia, 1997). En somme, le développement est un phénomène social. Et la "Gaani" se présente comme un creuset socioculturel, un atout pour le développement du pays Batonu.

D'autres fêtes communautaires qui s'inscrivent dans le même ordre d'idée de développement. Il s'agit de : la fête de l'igname à Savalou, le Nonvitcha, le Tolikounkanxwé, xэgbonuxwé, Gléxwéxwé, Adjaxwé, zèxwé, Alladaxwé, Festival de Danxome, etc.

En effet, selon les travaux de terrain, la fête de la "nouvelle igname" de Savalou est l'une des plus anciennes fêtes communautaires. Le 15 août de chaque année, la ville de Savalou célèbre la fête de l'igname: une fête coutumière qui prend de plus en plus d'ampleur. A l'origine, le peuple savalois organise cette fête pour remercier la providence pour l'abondance de la récolte. Selon Tossoh Gbaguidi XIII, roi de la localité, la spécificité de cette fête réside dans le fait que c'est l'espèce «Laboco » qui est célébrée, car c'est le tout premier tubercule qui est récolté. Cette fête de la nouvelle igname devenue une rencontre touristique est aussi une occasion pour le peuple "Mahi" de Savalou (groupe socio-ethnique basé dans le centre du bénin) de vouer un culte aux ancêtres pour avoir favorisé la bonne saison qui a permis la bonne récolté de l'igname. Pour agrémenter cette réjouissance, le peuple de Savalou organise plusieurs activités traditionnelles, que sont les différents cultes et prières publics animés de chants, louanges et danses culturelles. Chaque édition de cette fête draine de nombreux touristiques et curieux, tant nationaux qu'internationaux. Tout ceci génère beaucoup de 
devises pour la commune de Savalou. C'est une occasion de réjouissance et un atout pour le développement de la localité.

S'agissant de la fête "Nonvitcha', elle avait été créée en 1921 par la diaspora pour faire régner la fraternité au sein des communautés Xwla et Xwéda de la ville de Grand-Popo. Elle est célébrée tous les ans à la pentecôte. Aujourd'hui devenu un événement national. La grande fête se passe à la plage où les amis se réunissent pour manger et boire. Cette association avait pour principaux buts d'entretenir et resserrer les liens naturels de fraternité et de solidarité qui unissent originairement les Xwla et les Xwéda de toute obédience et de toute confession religieuse, de faire revivre les valeurs spirituelles, artistiques, folkloriques et historiques propres aux deux communautés sœurs et de définir et réaliser un programme de développement économique, social et culturel pour assurer un progrès réel de la population dans tous les secteurs.

En somme, à l'instar de la fête ''Wémcxwé', toutes les autres fêtes communautaires sur le plan national prônent le développement des localités où elles créées.

Quant aux autres fêtes communautaires, le Tolikounkanxwé, xગgbonuxwé, Gléxwéxwé, Adjaxwé, zèxwé, Alladaxwé, Festival de Danxoms, etc., ce sont des nouveaux creusets de retrouvailles dont le leitmotiv commun est la promotion du développement local.

De ce tout ce qui précède, les fêtes communautaires sont incontestablement un levier de développement dans les localités où elles sont créées. Elles accompagnent parallèlement le processus de décentralisation dans ses objectifs de développement local, par le fait qu'elles font participer les populations aux affaires de développement socio-économique de leur localité.

Cependant, l'évolution de ces différentes fêtes communautaires souffre énormément de leurs propres faiblesses et sont aussi confrontées à de nombreuses menaces qui détruisent les nobles initiatives de développement qu'elles portent.

En effet, les pesanteurs socio-culturelles sont les principales faiblesses qui inhibent les actions de développement de fête "Wémexwé". L'immixtion de la «politique » dans la gestion des affaires socio-culturelles constitue l'une des grandes menaces auxquelles elle est confrontée ; laquelle menace se manifeste par la lutte de leadership, les divisions intestines entre les membres du comité d'organisation de la fête. Aussi, les organisateurs de 'Wémexwé', se sont assignés en justice cette année 2017 en raison des mésententes récurrentes qui sont soldées par la division et la formation de deux camps qui s'affrontent pour des intérêts pécuniaires. Cet état de chose est aussi la triste réalité de la fête Nonvitcha, de zèxwé, de Alladaxwé, même si ces dernières n'ont pas encore des assignations en justice. 
En dépit de toutes les faiblesses et les menaces, ces fêtes communautaires participent, dans une large mesure, au développement local dans les communes où elles se célèbrent.

\section{Conclusion}

Cette étude a permis d'analyser le modèle de processus de développement local axé sur les réalités socio-culturelles des communes du Bénin. En effet, après une douzaine d'années d'expérimentation de la réforme de l'administration territoriale, l'étude de la problématique du développement local met à nu les faiblesses et les difficultés du processus de la décentralisation à assurer un véritable développement des collectivités locales.

Eu égard de tout ce qui précède et parallèlement au processus de la décentralisation, les populations locales mettent en place des initiatives endogènes dont les fêtes communautaires pour prendre en charge le développement de leur terroir.

L'étude de cas de la fête "Wémexwé" témoigne fort de l'importance capitale des fêtes communautaires dans le développement des localités. Alors initiée sur un fond de spiritualité liée à la mémoire du Révérend Père Dominique Adéyèmi qui avait impacté, de façon historique, le développement de la basse vallée de l'Ouémé pendant plus d'une trentaine d'années par le passé, cette fête devient aujourd'hui l'une des plus grandes institutions socio-culturelles du Bénin et un levier un véritable développement des communes de la Basse Vallée de l'Ouémé.

En définitive, l'importance des dynamiques endogènes des fêtes communautaires dans le développement local, parallèlement au processus de la décentralisation, démontre que le développement est d'abord culturel avant d'être politique. Cependant l'interaction de ces dynamiques socioculturelles et le processus de la décentralisation pourrait-elle constituer une source de conflit entre ces deux systèmes de voies de développement local, dans la mesure où les dynamiques endogènes concrétisent plus d'actions de développement à la base que le processus de la décentralisation qui peine encore à s'affirmer dans ce domaine ? De cette interrogation découlera le thème d'une prochaine étude sur les rapports entre les dynamiques endogènes par les fêtes communautaires et le processus de la décentralisation.

\section{References:}

1. Akande Aristide (2006). La stratégie de mise en ceuvre de la gouvernance locale pour un développement de la basse vallée de l'Ouémé. Travaux de Recherche action. P. 32. 
2. Deberre Jean-Christophe (2007). Décentralisation et développement local. Article Afrique contemporaine (n`221), p 45-54.

3. Dubresson André et al. (2005). Décentralisation et Développement local : un lien à repenser. Revue Tiers-Monde. P. 26.

4. Durkheim Emile (1894). Les règles de la méthode sociologique, PUF, Paris, France. 240 p.

5. Lafia Foulérath Bèrèkègui (1997). Aspects socio-culturels du développement dans la "Gaani"' cas de Nikki. Mémoire de maîtrise en Sociologie-Anthropologie. UNB-FLASH. 119 pages.

6. Mauss Marcel (1995). Sociologie-Anthropologie, Edition PUF. P. 26.

7. Olivier De Sardan Jean-Pierre (1995). Anthropologie et développement. Edition Karthala. Paris.

8. Olivier De Sardan Jean-Pierre \& Mohaman Tidjani Alou (2009). Les pouvoirs locaux au Niger. Tome 1 : A la veille de la décentralisation. Collection Voix d'Afrique. CODESRIA-Karthala. P.8.

9. Piveteau Alain (2005). Décentralisation et Développement local au Sénégal. Chronique d'un couple hypothétique. Revue Tiers-Monde. P. 18.

10. Soede Pascaline (2012). Impacts sociaux, économiques et environnementaux des inondations sur le développement de la commune d'Athiémè. Mémoire de maîtrise professionnelle en Développement communautaire. INJEPS-UAC. 84 pages.

11. Totte Marc et al. (2003). La décentralisation en Afrique de l'ouest : entre politique et développement. Cota-Karthala-Endra Grad, 403p

12. Zannou Sandé (2014). Gouvernance locale et stratégies de développement dans les communes $d u$ département du plateau au sud-est du Bénin. Thèse de Doctorat unique. FLASH/UAC. P. 11-28. 